\title{
Immature Platelet
}

National Cancer Institute

\section{Source}

National Cancer Institute. Immature Platelet. NCI Thesaurus. Code C13126.

A young platelet that contains residual mRNA and rRNA when released from the bone marrow into the peripheral circulation as a result of thrombopoiesis. Immature platelets normally make up a small percentage of the total circulating platelets (1.1-6.1\%, with a mean of 3.4\%.). An increased proportion of immature platelets in blood indicates increased thrombopoiesis. The relationship between the percent of immature platelets and the platelet count can be used to determine the rate of platelet turnover. 